\title{
Capillary electrophoretic separation of nanoparticles
}

\author{
Slawomir Oszwaldowski • \\ Katarzyna Zawistowska-Gibula $\cdot$ Kenneth P. Roberts
}

Received: 11 October 2010 /Revised: 22 November 2010 / Accepted: 2 January 2011 /Published online: 26 January 2011

(C) The Author(s) 2011. This article is published with open access at SpringerLink.com

\begin{abstract}
In the present work, CdSe nanocrystals (NCs) synthesized with a trioctylphosphine surface passivation layer were modified using amphiphilic molecules to form a surface bilayer capable of providing stable NCs aqueous solutions. Such modified nanocrystals were used as a test solute in order to analyze new electrophoretic phenomena, by applying a micellar plug as a separation tool for discriminating nanocrystals between micellar and micellefree zones during electrophoresis. The distribution of NCs between both zones depended on the affinity of nanocrystals towards the micellar zone, and this relies on the kind of surface ligands attached to the NCs, as well as electrophoretic conditions applied. In this case, the NCs that migrated within a micellar zone can be focused using a preconcentration mechanism. By modifying electrophoretic conditions, NCs were forced to migrate outside the micellar zone in the form of a typical CZE peak. In this situation, a two-order difference in separation efficiencies, in terms of theoretical plates, was observed between focused NCs $(\mathrm{N} \sim$ $10^{7}$ ) and a typical CZE peak for NCs $\left(\mathrm{N} \sim 10^{5}\right)$. By applying the amino-functionalized NCs the preconcentration of NCs, using a micellar plug, was examined, with the
\end{abstract}

Electronic supplementary material The online version of this article (doi:10.1007/s00216-011-4650-y) contains supplementary material, which is available to authorized users.

S. Oszwałdowski $(\bowtie) \cdot K$. Zawistowska-Gibuła

Faculty of Chemistry, Department of Analytical Chemistry,

Warsaw University of Technology,

ul. Noakowskiego 3,

00-664 Warsaw, Poland

e-mail: slaosw@ch.pw.edu.pl

K. P. Roberts

Department of Chemistry and Biochemistry,

The University of Tulsa,

Tulsa, OK 74104, USA conclusion that preconcentration efficiency, in terms of the enhancement factor for peak height $\left(\mathrm{SEF}_{\text {height }}\right)$ can be, at least 20. The distribution effect was applied to separate $\mathrm{CdSe} / \mathrm{ZnS}$ NCs encapsulated in silica, as well as surfacemodified with DNA, which allows the estimation of the yield of conjugation of biologically active molecules to a particle surface.

Keywords Capillary electrophoresis · Micelles . Nanocrystals · Focusing · Oligomer

\section{Introduction}

The recent interest in highly luminescent semiconductor nanocrystals has led to immense growth in their use in various applications such as bioimaging and immunoassays [1-5]. However, there is still a need for analytical methods that can provide quantitative and qualitative characterizations of nanostructure surface modifications (e.g., bioconjugation), commonly used in such assays. Although electron/scanning probe microscopies and X-ray/neutron scattering techniques have been applied to determine nanocrystal (NCs) core or core/shell dimensions [6], analytical methods for characterizing the nanocrystals surface and the degree of surface modification is deeply limited. Solution-based techniques such as fluorescence and Raman spectroscopies, due to a core complicate spectral effects [7] and possible energy transfer [4, 5, 8], as well as gel electrophoresis [9-11] have been shown to provide only semi-quantitative results. On the other hand, capillary electrophoresis (CE) has recently been demonstrated to have a potentially large utility in the quantitative separation of nanocrystalline structures (reviews: [12-15]). Although the separation mechanism for nanoparticles is less linear 
than for traditional molecular and ionic CE solutes, the intrinsic hydrodynamic and electrophoretic properties of nanoparticles, before and after surface modification, have been shown proved to be an affordable tool for the characterization of nanostructures $[16,17]$. In this context, the quantitative relationship between a width of CE peak and a size distribution of particular particles [16], the interaction between two nanostructures functionalized with biological ligands [18], the determination of NCs effective charge and concentration by means of capillary isotachopheresis [19], as well as the reversed electrode polarity stacking method of CE, with the use of anionic surfactant, for enrichment of Au nanoparticles [14, 20-22] have been reported recently.

It is generally accepted that electrophoretic migration of nanoparticles is much more complex phenomenon than migration of a typical solute. For a charged spherical particle dispersed in an electrolyte, the following forces have to be considered: (1) the electrostatic force exerted by the applied electric field, (2) Stokes friction, (3) electrophoretic retardation, and (4) relaxation effects $[12,16]$. Depending on particle size (speaking precisely, the value of $\kappa a$ the ratio of the radius of the core particle $(a)$ to the electrical double-layer thickness $\left(\kappa^{-1}\right)$ ), Helmholtz-Smoluchowski, Hückel-Onsager, or Henry's equation, respectively, should be applied for the characterization of the particles migration [23]. For particles at the nanoscale, the Henry's equation seems to be the most appropriate, however with the limitation that $\zeta$-potential should be $<25-50 \mathrm{mV}$ [23]. Many studies have substantiated that nanoparticles migration velocity is reliant on zeta potential and particle size $[12,13,16]$.

In the present work, the electrophoretic separation of nanoparticles coated with surfactant molecules was examined in order to develop a method suitable for separating a wide range of nanoparticles. Covering nanostructures with amphiphilic molecules was used to the functionalization of nanostructures, e.g., solubilization of nanocrystals [24-30], single-walled nanotubes [31-34], and fullerenes [35], and these were reviewed recently $[1,5]$. For these examples, anionic (e.g., sodium dodecyl sulfate (SDS), dioctylsulfosuccinate (DOSS)), cationic (CTAB), or non-ionic (Tritons) surfactants were used for coating, with the consequence of imposing a surface charge on the nanostructures and consequent electrostatic double-layer sufficient for the separation under aqueous electrophoretic conditions. Capillary electrophoretic methods [29, 30, 34, 35] were applied to characterize modified in this way nanostructures.

In order to overcome the limitations in the electrophoretic separation of nanoparticles, imposed by four forces mentioned above, the introduced recently to literature the separation tool-a micellar plug, has been examined. According to literature reports, the micellar plug was used as a preconcentration/separation tool for solute molecules
[36-41] and based on this tool a novel separation mechanism (analyte focusing by micelle collapse) was introduced recently to separation science [37]. However, in the case of nanoparticles, apart from the preliminary results [30], the tool has not been applied yet. In the present work it was found that, preconcentration of NCs within a micellar plug allows NCs to be focused with high separation efficiency $\left(N \sim 10^{7}-10^{8}\right)$. By modifying electrophoretic conditions, NCs were forced to exit the micellar plug and migrate, e.g., according to CZE mechanism, this with lower separation efficiency $\left(\mathrm{N} \sim 10^{5}\right)$. Note that focusing of volume solutes (bacteria) with ultrahigh $\mathrm{CE}$ separation efficiency $\left(\mathrm{N} \sim 10^{9}\right)$, presumably due to solute-solute interaction, was reported [13, 42].

In the present work, CdSe NCs, surface-modified with dihydrolipoic acid (DHLA), 1, 10-phenathroline derivative $\left(\mathrm{PhSO}_{3}\right)_{2}(\mathrm{NN})$, DNA oligomer, as well as commercial amineterminated nanoparticles as test solutes have been applied to examine the novel separation scheme. Secondly, the established mode of the separation was used to control the modification of surface of nanoparticles, where unmodified and modified nanoparticles were monitored simultaneously.

These results will be especially valuable in monitoring and controlling the conjugation of biologically active molecules to NC surfaces for improved bioassays.

\section{Experimental}

\section{Instrumentation}

The TEM instrument (Hitachi H 7000 (Hitachi, Japan)) was operated at $90 \mathrm{kV}$ with a $5 \%$ standard deviation in size determinations. Zetatrac and Nanotrac 151 Ultra (Microtrac, Inc., Montgomeryville, PA) particle size analyzers provided additional size determinations, as well as particles zeta potential. The standard deviation of the instrument in the measured size range was $\pm 2 \mathrm{~nm}$. Gel electrophoresis separation was performed with the use of PowerPac 200, Bio-Rad, Hercules, USA. After separations, gels were photographed under UV light using an Alpha Innotech FluorChem HD2 (San Leandro, USA).

All CE electropherograms were obtained using a P/ACE MDQ CE system from Beckman-Coulter Instruments (Fullerton, CA). System operation and data collection were controlled using Karat 5.0 software supplied by BeckmanCoulter Instruments. CE experiments were performed with the use of $75 \mu \mathrm{m}$ i.d. $\times 70 \mathrm{~cm}$ long fused-silica capillaries (Polymicro Technologies, Phoenix, AZ). The length from injector to detector was $60 \mathrm{~cm}$. The capillary was conditioned daily by rinsing for 15 min with $0.1 \mathrm{M} \mathrm{NaOH}$ followed by $3 \mathrm{~min}$ with water and $15 \mathrm{~min}$ with running buffer. Injection was performed hydrodynamically by 
applying $35 \mathrm{mbar}$ for $5 \mathrm{~s}$. The capillary was rinsed with $0.1 \mathrm{M} \mathrm{NaOH}$ for $5 \mathrm{~min}$ and running buffer for $5 \mathrm{~min}$ between runs. The separation capillary was temperature controlled to $25{ }^{\circ} \mathrm{C}$ by liquid cooling of the P/ACE MDQ instrument.

\section{Materials}

NCs surface-functionalized with a primary amine moiety (Maple Red-Orange T2-MP EviTags) were purchased from Evident Technologies (Troy, New York). The NCs were composed of cadmium selenide core structures coated with a zinc sulfide outer shell. The total core/shell size was 5$7 \mathrm{~nm}$ in diameter $\left(\lambda_{\mathrm{abs}}=590 \mathrm{~nm} ; \lambda_{\mathrm{em}}=620 \mathrm{~nm}, 11 \mathrm{nM}\right)$. According to the manufacturer, the surface of the nanocrystals contained a lipid polyethylene glycol coupled to amine groups to provide up to $30 \%$ coverage with the amine. The total hydrodynamic diameter in water was $\sim 25 \mathrm{~nm}$, according to manufacturer [43].

For the synthesis of NCs encapsulated in silica, commercially available CdSe/ZnS EviDots (surface passivated with trioctylphosphine (TOP) oxide (TOPO)) were utilized. The absorbance and emission maxima were 590 and $68 \mathrm{~nm}$, respectively, with a provided concentration of $1.5 \mathrm{mg} / \mathrm{mL}(16 \mathrm{nM})$ and a $4 \mathrm{~nm}$ core/shell diameter [43]. CdSe/ZnS NCs modified with DHLA were purchased from NANOCO (Manchester, UK) and provided in water at a concentration of $10 \mathrm{mg} / \mathrm{mL}$. The absorbance and emission maxima were 590 and $610 \mathrm{~nm}$, respectively.

The DNA oligomer, with a sequence of $5^{\prime}-\mathrm{HS}-\left(\mathrm{CH}_{2}\right)_{6-}$ GGCCACTAACATGGGGACCA-3', was provided at by Biosearch Technologies (Novato, CA). The DNA sample was diluted in $10 \mathrm{mM}$ borate buffer to a concentration of 250 nM. PD-10 columns-Sephadex G-25, as well as Superdex 200 column (GE Healtcare Bio-Sciences AB, Uppsala, Sweden). Immobilized tris(2-carboxyethyl)phosphine (TCEP) disulfide reducing gel and succinimidyl-4( $N$-maleimidomethyl)-cyclohexane-1-carboxylate (SMCC) (Pierce Biotechnology, Rockford, USA).

For the synthesis of the TOP coated CdSe nanoparticles, the cadmium oxide $(\sim 1 \mu \mathrm{m}, 99.5 \%)$, selenium powder (100 mesh, 99.999\%), trioctylphosphine (TOP, 90\%) from Sigma-Aldrich (St. Louis, MO) were used. Sodium tetraborate, $\left(\mathrm{PhSO}_{3}\right)_{2}(\mathrm{NN})$ (4,7-diphenyl-1,10-phenanthrolinedisulfonic acid disodium salt), tetraethyl orthosilicate, as well as surfactants: sodium dioctylsulfosuccinate (DOSS), sodium laurate (LA), sodium oleate (OA) and Triton X-100 (polyethylene(10) isoctylphenyl ether); poly(ethylene oxide) $\mathrm{M}_{\mathrm{v}} 8,000,000$ and 600,000, 100,000 were obtained from Sigma-Aldrich (St. Louis, MO). Silica compounds APTEC and THPMP used for NCs silica encapsulation were from Gelest (Morrisville, USA). Materials: Agarose, $1 \times$ Tris/borate/ EDTA (TBE) and solvents: chloroform, toluene, cyclohexane
(Fisher Scientific, USA). Amicon Ultra 15 centrifugal filter tubes (100,000 NMWL) and 18.2 $\mathrm{M} \Omega$ MilliporeWater (Millipore, Billerica USA) were used throughout the present work.

Bioconjugation of NCs with DNA oligomers

Conjugation of amine-EviTags or silica-encapsulated EviDots with DNA oligomer was carried out according to protocols provided by the manufacturer [43]. A 2 nanomole of NCs was activated using succinimidyl-4-( $N$-maleimidomethyl)cyclohexane-1-carboxylate (SMCC). Size exclusion chromatography (SEC) using a PD-10 column was used to remove excess SMCC. Immobilized TCEP disulfide reducing gel was added to the oligomer solution to expose free - $\mathrm{SH}$ groups and then removed by centrifugation. Activated NCs were covalently attached to the reduced aptamer by combining both solutions for $4 \mathrm{~h}$ (or overnight). The NC-oligomer conjugate was centrifuged through Amicon Microsep 100,000 spin filters at 3,000 rpm to concentrate the samples prior to final purification by SEC using Superdex 200.

\section{Preparation of $\mathrm{CdSe} / \mathrm{ZnS} /$ silica functionalized NCs}

The silica encapsulation process utilized hydrophobic $\mathrm{CdSe} /$ $\mathrm{ZnS}$ EviDots as a base substrate (manuscript in preparation). In brief (Fig. S8 in the Electronic supplementary material), hydrophobic EviDots were transferred from toluene to water through the formation of a surfactant/lipid bilayer on the NCs surface. A microemulsion was then prepared by addition of the water soluble nanostructures to cyclohexane. The addition of tetraethyl orthosilicate and base initiates silica polymerization on the NCs surface. Toward the end of the polymerization, 3-aminopropyltriethoxysilane and 3-(trihydroxysilyl)propyl methylphosphonate, monosodium salt were introduced into the emulsion to provide water solubility and surface reactivity. In order to obtain aptamer derivative ( $\mathrm{CdSe} / \mathrm{ZnS} /$ silica/aptamer), the procedure presented above was applied.

\section{TEM sizing of silica-encapsulated CdSe/ZnS NCs}

One drop of a dilute sample of NCs in water solution was placed onto a Formvar-coated copper grid and allowed to settle for $20 \mathrm{~s}$ and wicked away using an absorbent tissue. Size analysis was performed on captured digital images using ImageJ V. $1.34 \mathrm{~s}$ program.

Agarose gel electrophoresis

Separation of NCs before and after oligomer conjugation was conducted using previously reported agarose gel electrophoresis conditions [9-11]. Samples were run on a 
$1 \%$ agarose gel in $1 \times$ TBE for $60-70$ min using the BioRad PowerPac 200 apparatus set at a constant voltage of $90 \mathrm{~V}$. The gels were photographed under UV light using an Alpha Innotech FluorChem HD2.

\section{Capillary electrophoresis separation}

For the preliminary experiments a micellar plug containing $\mathrm{CdSe} / \mathrm{TOP} / / \mathrm{LA} \mathrm{NCs}$, obtained by dispersing CdSe/TOP NCs in TX-100/LA solution was used. Other samples prepared for CE experiments were obtained by adding, up to $10 \mu \mathrm{L}$ of mixture of TX-100 and DOSS solution to a $10 \mu \mathrm{L}$ solution of functionalized and non-functionalized nanostructures (CdSe NCs, CdSe/ZnS NCs, CdSe/ZnS// silica shell nanoparticles (NPs)). Sample solutions were injected into the capillary from the anode side with $35 \mathrm{mbar}$ for $5 \mathrm{~s}$. Separation buffers contained $10 \mathrm{mM}$ sodium tetraborate were applied. The buffer was modified (typically: 5 or $10 \mathrm{mM}$ sodium tetraborate, $\mathrm{pH}$ 9.4) by adding methanol $(\mathrm{MeOH})$ or acetonitrile $(\mathrm{ACN})$ in the range up to $40 \%$ (v:v). Details of each separation buffer composition are specified in the figure captions. Separations were conducted using voltages in the range of 3-25 kV. Peak parameters (peak area, height, width, as well as peak spectrum) were obtained using the instrument software.

\section{Results and discussion}

Capillary electrophoresis of CdSe NCs coated with amphiphilic molecules

In the present work CdSe NCs passivated by surface TOP ligands were synthesized and characterized. By dispersing such NCs in aqueous solution of anionic amphiphiles, the aqueous soluble, an amphiphile-coated nanocrystals, in the form of $\mathrm{CdSe} / \mathrm{TOP} / /$ amphiphile NCs were obtained. Both procedures (synthesis and modification of CdSe nanocrystals) and basic characterization of such structures were included in the Electronic supplementary material along with Fig. S1.

Either CZE or MEKC capillary electrophoretic mode was reported to be applied to visualize peak for $\mathrm{CdSe} /$ amphiphile NCs [30], and these modes were applied here toward $\mathrm{CdSe} / \mathrm{TOP} / / \mathrm{LA} \mathrm{NCs}$ (Fig. 1(frames a, c)). An interesting situation was obtained by introducing a micellar plug of non-ionic surfactant for mobilizing $\mathrm{CdSe} / \mathrm{TOP} / \mathrm{LA}$ NCs. In this case, a new peak for $\mathrm{CdSe} / \mathrm{TOP} / /$ amphiphile $\mathrm{NCs}$ was observed, regardless of CE separation mode applied (Fig. 1(frames b, d)). This issue was additionally confirmed using only CZE mode, to avoid additional equilibria due to MEKC mode (Fig. 2). In this case, $\mathrm{CdSe} / \mathrm{TOP} / / \mathrm{LA} \mathrm{NCs}$ were visualized as CZE peak (frame a),

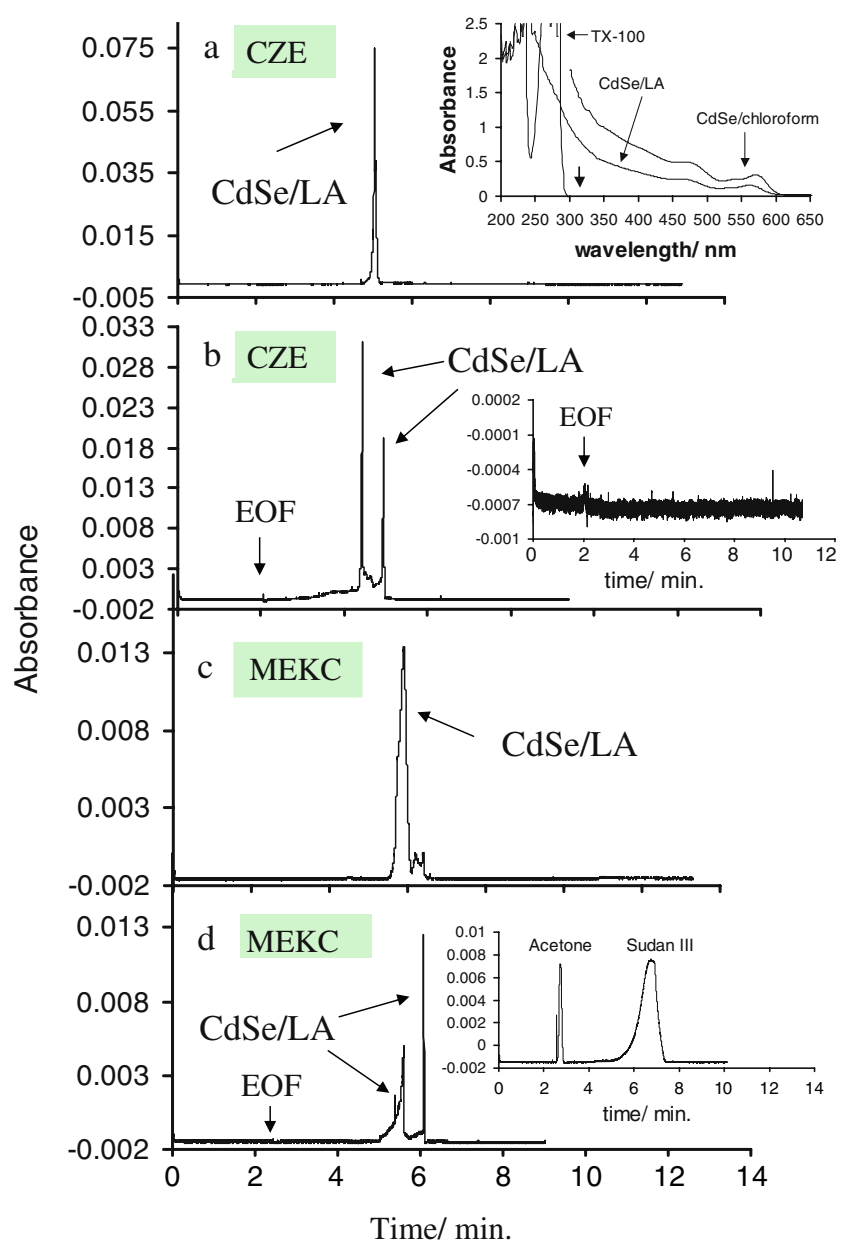

Fig. 1 Effect of the addition of the non-ionic surfactant (TX-100) to the sample of CdSe/TOP//LA NCs. Sample analyzed: CdSe NCs coated with sodium laurate; nanocrystal size, $3.4 \mathrm{~nm}$ (sample preparation, CdSe NCs dispersed in $200 \mathrm{mM}$ sodium laurate). $a-b$ $C Z E$ mode, electrolyte buffer, $20 \mathrm{mM}$ sodium tetraborate; $c-d, M E K C$ mode, electrolyte buffer, $50 \mathrm{mM}$ SDS containing $10 \mathrm{mM}$ sodium tetraborate. $a, c$ Only CdSe NCs coated with sodium laurate; $b, d$ Same samples containing the non-ionic surfactant (TX-100); $b 6 \%$ and $d 8 \%$ TX-100, respectively. Voltage applied: $a, b+25 \mathrm{kV}(77 \mu \mathrm{A})$ and $c, d+20 \mathrm{kV}(42 \mu \mathrm{A})$. Detection: UV-vis, $\lambda=330 \mathrm{~nm}$. Sample injection: $50 \mathrm{mbar} / 0.3 \mathrm{~min}$. $a$ (inset) Spectra for TX-100, as well as for NCs uncoated and coated with sodium laurate, respectively. The vertical arrow shows the detection choice, selective for NCs $(\lambda=$ $330 \mathrm{~nm})$. The detection wavelength $(\lambda=330 \mathrm{~nm})$ was checked applying a blank sample (200 mM sodium laurate/10\% TX-100) $(b$ (inset)). Finally, $d$ (inset) is the MEKC separation of a pair of markers at the MEKC conditions applied here

focused peak (frame c) or as both peaks simultaneously (frame b). There is two order difference in separation efficiencies, in terms of theoretical numbers of plates $(N=$ $\left.5.54\left[\mathrm{t}_{\mathrm{i}} /\left(\text { peak area } \mathrm{i} / \text { peak height }_{\mathrm{i}}\right)\right]^{2},[44]\right)$, between CZE peak (frame a) and focused NCs (frame c), which is a direct evidence that both peaks were obtained due to different separation mechanisms - this despite the use of the same CE mode (CZE) applied to run the sample. 


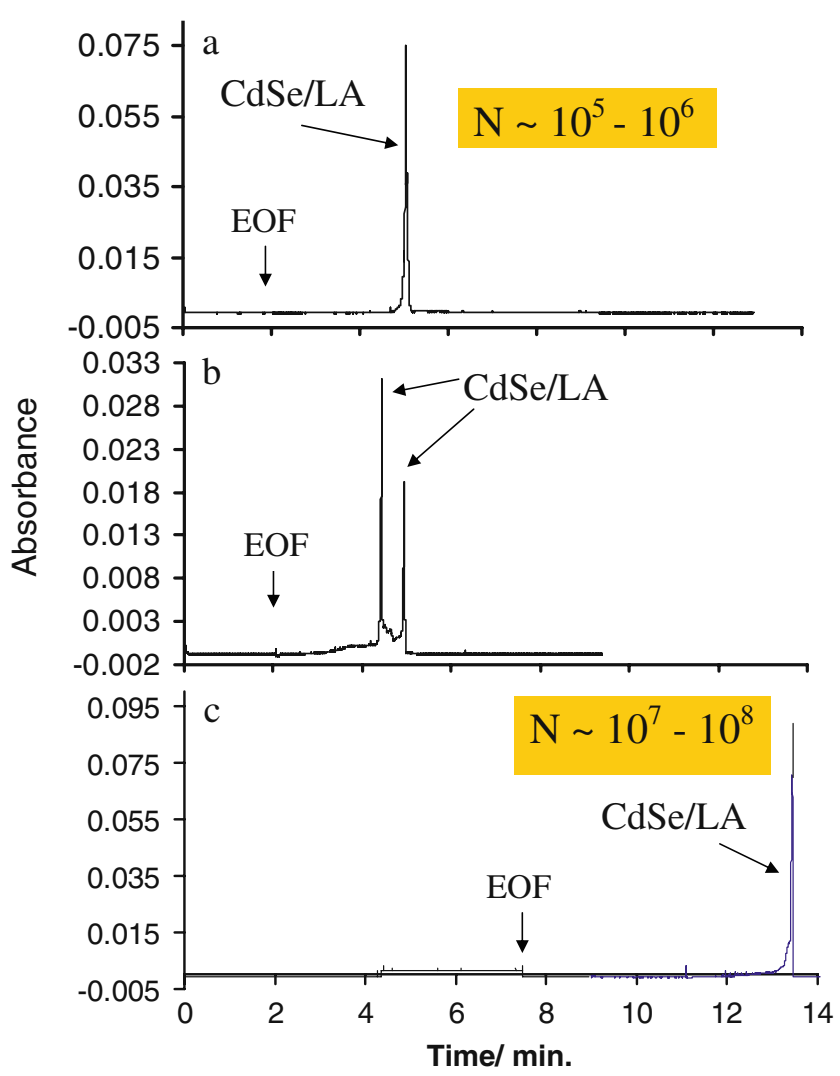

Fig. 2 Effect of concentration of the non-ionic surfactant (TX-100) in the plug on the form of peaks for CdSe/TOP//LA NCs. Sample: CdSe/ TOP NCs dispersed in $0.2 \mathrm{M}$ LA containing: $a 0.5 \%$ TX-100 (only CZE peak), $b 3 \%$ TX-100 (both CZE and focused peaks), and $c 10 \%$ TX-100 (only focused peak). Separation conditions: electrolyte buffer $10 \mathrm{mM}$ sodium tetraborate, applied voltage $+20 \mathrm{kV}$ (measured current $40 \mu \mathrm{A}$ ), detection $\lambda=330 \mathrm{~nm}$. Sample injection: $50 \mathrm{mbar} / 0.3 \mathrm{~min}$. $\mathrm{CdSe} / \mathrm{TOP} / / \mathrm{LA}$ QDs size, $3.4 \mathrm{~nm}$

Specifically, CZE electropherograms for focused NCs were recorded at two wavelengths of UV-vis detector (Fig. 3), thus at $\lambda=330 \mathrm{~nm}$, selective for only NCs and at $\lambda=245 \mathrm{~nm}$ for both components, micellar plug and NCs, respectively (see Fig. 3(inset)). The obtained picture shows (Fig. 3) that $\mathrm{CdSe} / \mathrm{TOP} / / \mathrm{LA} \mathrm{NCs,} \mathrm{in} \mathrm{the} \mathrm{form} \mathrm{of} \mathrm{a} \mathrm{narrow}$ zone, were located inside a broader micellar plug, at the boundary between micellar and micelle-free zone (frame b). This denotes a preconcentration mechanism in this situation.

It was established that the electrophoretic conditions used to obtain focused peak for $\mathrm{CdSe} / \mathrm{TOP} / /$ amphiphile NCs preclude separation of two NCs of different dimensions or coated with different surfactants and only one cumulative peak was seen at the border between micellar and micelle-free zone. In order to overcome this limitation, composition of either an electrolyte buffer or a micellar plug was modified either by changing an ionic strength or by adding organic solvents. Both ways allow $\mathrm{CdSe} / \mathrm{TOP} /$ amphiphile NCs to be released from micellar plug to electrolyte buffer, to form a new peak (Fig. 4); with the most important remark, that the effect can be controlled by current electrophoretic conditions. Obviously, in this case, when NCs passing the boundary micellar/micelle-free zone, the colloidal particles are destabilized, which leads to a decrease in separation efficiency $\left(N \sim 10^{5}-10^{6}\right)$ (Fig. 4). This is similar level of $\mathrm{N}$ to this reported for the CZE separation of charged nanoparticles [17].

Electrophoretic phenomena for $\mathrm{CdSe} / \mathrm{TOP} / /$ amphiphile - a comment

Functionalization of CdSe NCs using ionic amphiphilic molecules allows introduce electrostatic double-layer sufficient for separating NCs under aqueous conditions. However, specifically for amphiphile-coated NCs a few additional issues should be discussed in order to explain electrophoretic results presented in the section above (Figs. 1-3). Firstly, based on results of the previous work [30], the co-migration of micellar aggregates with amphiphile-coated CdSe NCs, irrespective of an amphiphile applied (ionic or non-ionic), was observed (MEKC). This was additionally confirmed in the present work (Fig. 1). However, the co-migration of aqueous soluble NCs with micellar aggregates (detected by hydrophobic marker-Sudan III) is a contradiction. The shift in NCs migration means that there is a significant interaction between NCs and micellar aggregates enforced by structural and behavioral similarity between surfactant-coated NCs and regular micelles (in terms of size, the presence of charged or polar surface groups). This forces NCs to be incorporated into the zone of micellar aggregates, which means that those NCs can be regarded as pseudomicelles, with higher affinity toward a zone of micellar aggregates than toward a micellefree zone. This means that pseudomicelles did form a stable mixed pseudomicellar phase with regular micelles and under this state NCs could be manipulated in terms of appropriate electrophoretic phenomena (transport, accumulation, and release).

Secondly, apart from this aspect, the migration mechanism using a micellar plug as a separation tool is necessary to discuss in order to analyse results above (Figs. 1-3). Recently, the tool was introduced to the separation science and used to separate or preconcentrate variety of solutes [36-41]. Taking both issues into account (formation of mixed pseudomicellar system and the use a micellar plug as a separation tool) it can be stated that focusing of NCs is due the preconcentration mechanism, based on the formation of mixed pseudomicellar system. Modifying electrophoretic conditions (Fig. 4), the controllable release of CdSe/TOP//amphiphile NCs from micellar plug to electrolyte buffer was observed and this effect formally means the demixing of a narrow zone of mixed pseudomicelles at the micelle/micelle-free boundary (denoted as MDZ, [37]). The released from micellar plug NCs were found to migrate 
Fig. 3 Visualization of $\mathrm{CdSe} /$ TOP//LA NCs being in micellar plug by using two wavelengths of the detector. Sample: CdSe/ TOP NCs dispersed in $10 \% \mathrm{w} /$ $w$ TX-100 containing 0.2 M LA. Blank sample: $10 \% w / w$ TX-100 containing 0.2 M LA. $a$ Detection at $330 \mathrm{~nm}$ (only peak of focused NCs); $b$ detection at $245 \mathrm{~nm}$ (both micellar plug and focused NCs); $c$ detection at $245 \mathrm{~nm}$ (micellar plug $\rightarrow$ injection of a blank sample only). Peaks $A$ CdSe/TOP//LA NCs; $B$ micellar plug (TX-100/LA). Separation conditions: running buffer $10 \mathrm{mM}$ sodium tetraborate, voltage $+20 \mathrm{kV}$. Sample injection: $50 \mathrm{mbar} / 0.3 \mathrm{~min}$. Absorbance axis of each frame is set at the same level in order to show peaks proportions. $a$ (inset) The spectra for TX-100 and $\mathrm{CdSe} / \mathrm{TOP} / / \mathrm{LA} \mathrm{NCs}$, respectively. Vertical arrows show wavelengths applied to UV-vis detector of the CE set

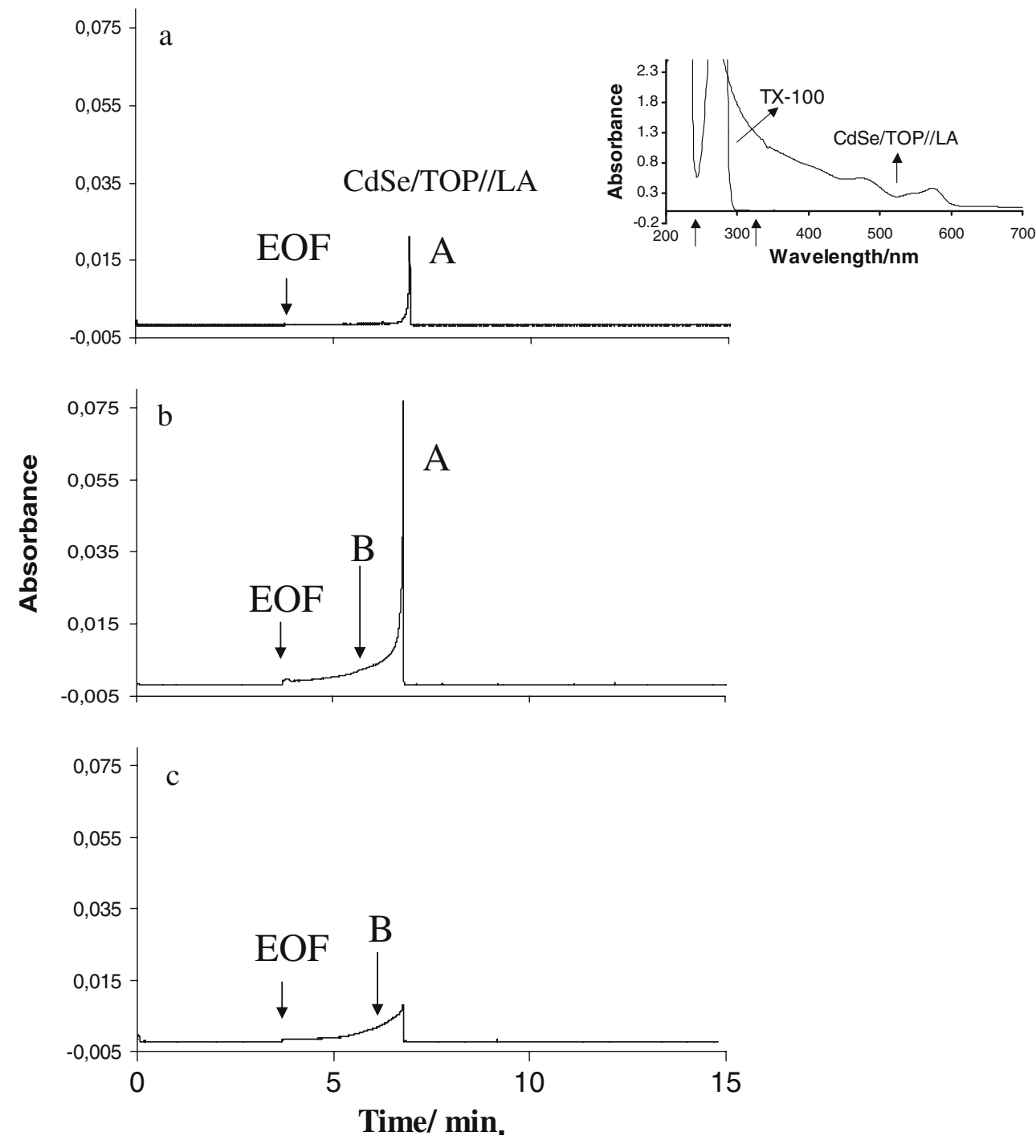

according to, e.g., CZE mode. The meaning of statements above is, that applying the micellar plug for $\mathrm{NCs}$, the electrophoretic mechanism can be switched from one mechanism (NCs preconcentration in a micellar plug) to another (CZE mode for NCs migrated outside a micellar plug), as well as two mechanisms were available simultaneously, under the same run (e.g., Figs. 2 b or 4b). The latest remark is in agreement with Quirino [38], where double mechanism on the same run, using a micellar plug, was reported.

Due to the importance of phenomena discussed above, the present section was extended, in the form of a more detailed discussion included to the Electronic supplementary material, along with Figs. S2-S4 in the Electronic supplementary material. A wider discussion on the abovementioned mechanism is related with Fig. S4 in the Electronic supplementary material). Also, within this section two issues were highlighted. Firstly, it was indicated that the modification of an electrolyte buffer proved to be the most effective way for releasing CdSe/TOP//amphiphile NCs from a micellar plug, in contrast to modification of a micellar plug alone (Fig. S2 in the Electronic supplementary material). This additionally confirms that demixing of a narrow zone of mixed pseudomicelles found between a micellar plug and an electrolyte buffer is the key factor for NCs exit. This way of modification was applied in sections below, where NCs exit a micellar plug was used as a tool for NCs discrimination. Secondly, Fig. S3 in the Electronic supplementary material shows that applying various voltages, each NCs peak (focused NCs vs. CZE peak for NCs) responses differently in terms of peaks shape, which additionally confirms the occurrence of double mechanisms, on the single electrophoretic run.

The last issue worth discussing is the shape similarity between focused NCs (sharp peak) and a peak (generally for particles) called in literature "spikes" [12]. These are sharp peaks with no reproducible migration, due to the presence of small aggregates. However, the observed in the 
Fig. 4 Effect of the electrolyte buffer modification on distribution of CdSe/TOP NCs coated with the LA surfactant. $a$ Peak of $\mathrm{CdSe} / \mathrm{TOP} / / \mathrm{LA} \mathrm{NCs}$ in micellar plug. $b \mathrm{CdSe} / \mathrm{TOP} / / \mathrm{LA}$ being in the micellar plug and the electrolyte buffer zone simultaneously. $c$ Peak of CdSe/ $\mathrm{TOP} / / \mathrm{LA}$ in electrolyte buffer zone. Sample preparation: $\mathrm{CdSe} /$ TOP NCs, core size $2.9 \mathrm{~nm}$, dispersed in mixture of $0.5 \mathrm{ml}$ $10 \% \mathrm{w} / \mathrm{w}$ TX-100 containing 0.2 M LA. Separation conditions: $a 10 \mathrm{mM}$ sodium tetraborate; $b 10 \mathrm{mM}$ sodium tetraborate $/ 20 \% \mathrm{ACN} ;$ c $10 \mathrm{mM}$ sodium tetraborate $/ 30 \% \mathrm{ACN}$; all $+20 \mathrm{kV}$. All peaks were detected at $\lambda=330 \mathrm{~nm}$, selective for NCs. In this situation (peak in micellar plug vs. peak in electrolyte buffer zone), $\mathrm{SEF}_{\text {height }}$ was equal to 7 . Each frame is set at the same AU (axis) to show peaks height proportions. In addition, each of the situation is graphically illustrated to distinguish migration phenomena, where yellow zone is the micellar plug (TX-100/ LA) and red zone is the NCs zone of $\mathrm{CdSe} / \mathrm{TOP} / / \mathrm{LA} \mathrm{NCs}$

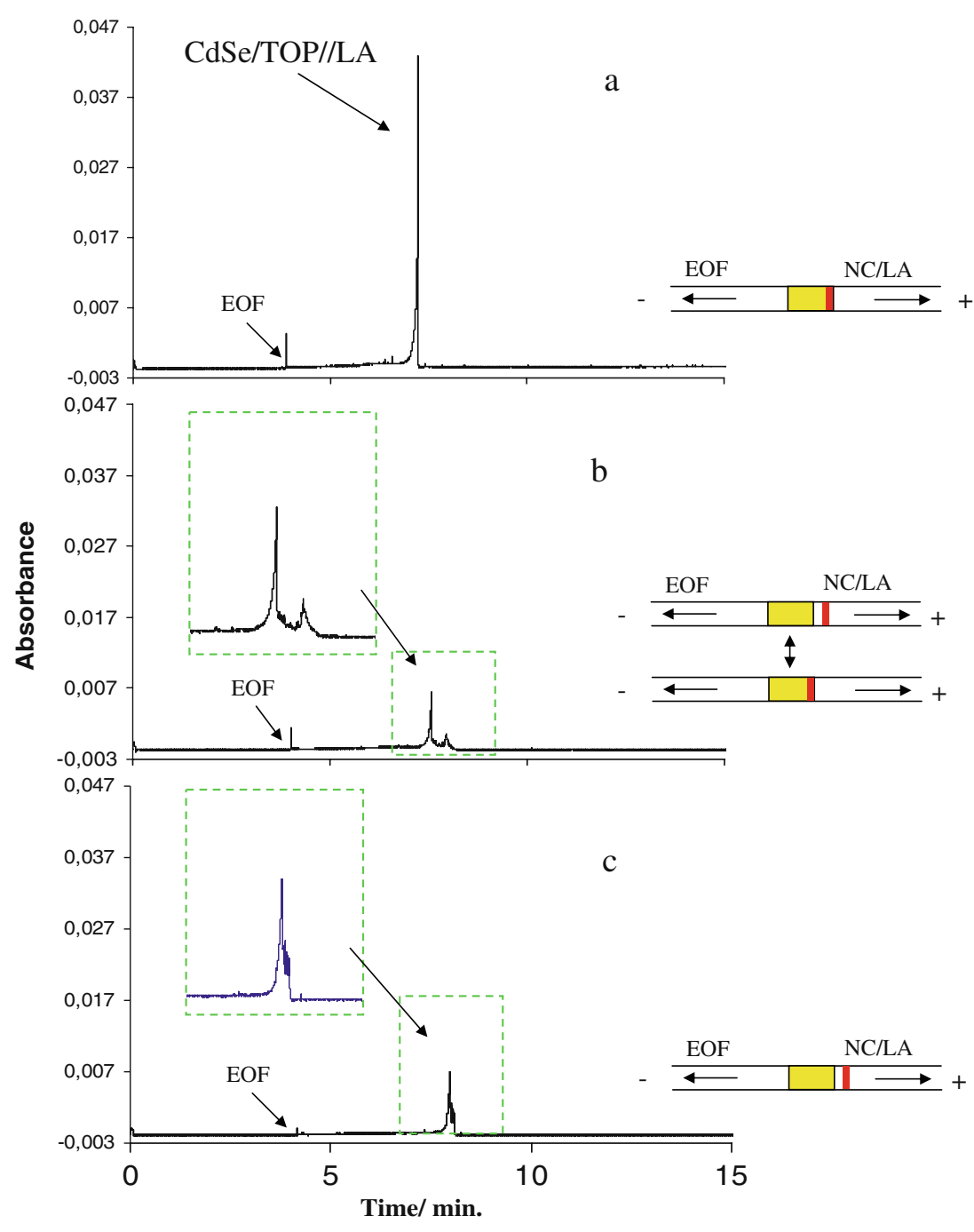

present work, a sharp peak for focused NCs is definitely not a spike as the peak position proved to be reproducible and can be controlled by current electrophoretic conditions. Also, additional conditions applied, in order to remove aggregates from the sample (centrifugation, ultrasonification) did not change an electropherogram, that a sharp peak for NCs within a micellar plug (focusing) was seen, generally without major changes.

Capillary electrophoresis of NCs modified with bidentate surface ligands

In this section, the focusing effect was examined for $\mathrm{CdSe} /$ $\mathrm{ZnS}$ NCs coated with anionic SDS surfactant, as well as $\mathrm{CdSe} / \mathrm{ZnS}$ NCs passivated by negatively charged bidentate ligands (Scheme S1 in the Electronic supplementary materials). The purpose of this experiment was to demonstrate that $\mathrm{CE}$ phenomena discussed in both Sections above are suitable for manipulating peaks of NCs others than those coated with surfactants. Bidentate ligands were chosen due to their common use in NCs surface functionalization and their ability to stabilize (NC ligand) system and provide water solubility of the final product. Commercially available core/shell (CdSe/ZnS) NCs passivated by TOPO or DHLA ligands were used in the experiments, below (Fig. 5).

Shown in Fig. 5. (electropherograms a-c) are results for the separation of $\mathrm{NC}$ starting material, $\mathrm{A}-\mathrm{CdSe} / \mathrm{ZnS} /$ TOPO NCs, and its derivatized product, B-CdSe/ZnS/ DHLA. The mixture was prepared by adding solution of CdSe/DHLA to SDS surfactant solution containing CdSe/ $\mathrm{ZnS} / \mathrm{TOPO}$ to form (CdSe/ZnS/TOPO//SDS) and the final solution was then injected for CE separation. The separation buffer was $10 \mathrm{mM}$ sodium tetraborate with varying amounts of organic modifier, $\mathrm{ACN}$, to manipulate the selectivity of the separation. When no organic modifier was used, both NCs co-migrated within the micellar plug. Although, focusing of the NCs into a narrow zone was achieved, resolution of the two different NCs was not observed. With $10 \% \mathrm{ACN}$ in the separation buffer 
Fig. 5 Comparison of the separation of two couples of modified NCs with the use of micellar zone mode. Electropherograms: $a-c$ Mixture of $A$, $\mathrm{CdSe} / \mathrm{ZnS} / \mathrm{TOPO} / / \mathrm{SDS}$ and $B$, $\mathrm{CdSe} / \mathrm{ZnS} / \mathrm{DHLA}$. Electrolyte buffer: $a 10 \mathrm{mM}$ sodium tetraborate (both NCs in micellar zone); $b 10 \mathrm{mM}$ sodium borate/ $10 \%$ ACN (peak CdSe/ZnS/ $\mathrm{TOPO} / / \mathrm{SDS}$ in micellar zone, peak CdSe/ZnS/DHLA in buffer zone); c $10 \mathrm{mM}$ sodium borate/ $15 \%$ ACN (both NCs in buffer zone). Electropherograms: $d-f$ mixture of $A, \mathrm{CdSe} / \mathrm{ZnS} / \mathrm{TOPO} / /$ $\mathrm{SDS}$ and $C, \mathrm{CdSe} / \mathrm{ZnS} /$ $\left(\mathrm{PhSO}_{3}\right)_{2}(\mathrm{NN})$. Electrolyte buffer: $d 10 \mathrm{mM}$ sodium tetraborate (both NCs in micellar zone); $e$ $10 \mathrm{mM}$ sodium tetraborate $/ 15 \%$ ACN (peak CdSe/ZnS/ $\left(\mathrm{PhSO}_{3}\right)_{2}(\mathrm{NN})$ in micellar zone, peak CdSe/ZnS/TOPO//SDS in buffer zone); $f 10 \mathrm{mM}$ sodium tetraborate $/ 20 \% \mathrm{ACN}$ (both NCs in buffer zone). Peak of NCs was confirmed by the DAD spectrum of the appropriate peak, as shown in the example (f). Voltage: $+17 \mathrm{kV}$ for all examples. Detection wavelength: $a-e 293 \mathrm{~nm}$; $244 \mathrm{~nm} ; f$ $237 \mathrm{~nm}$
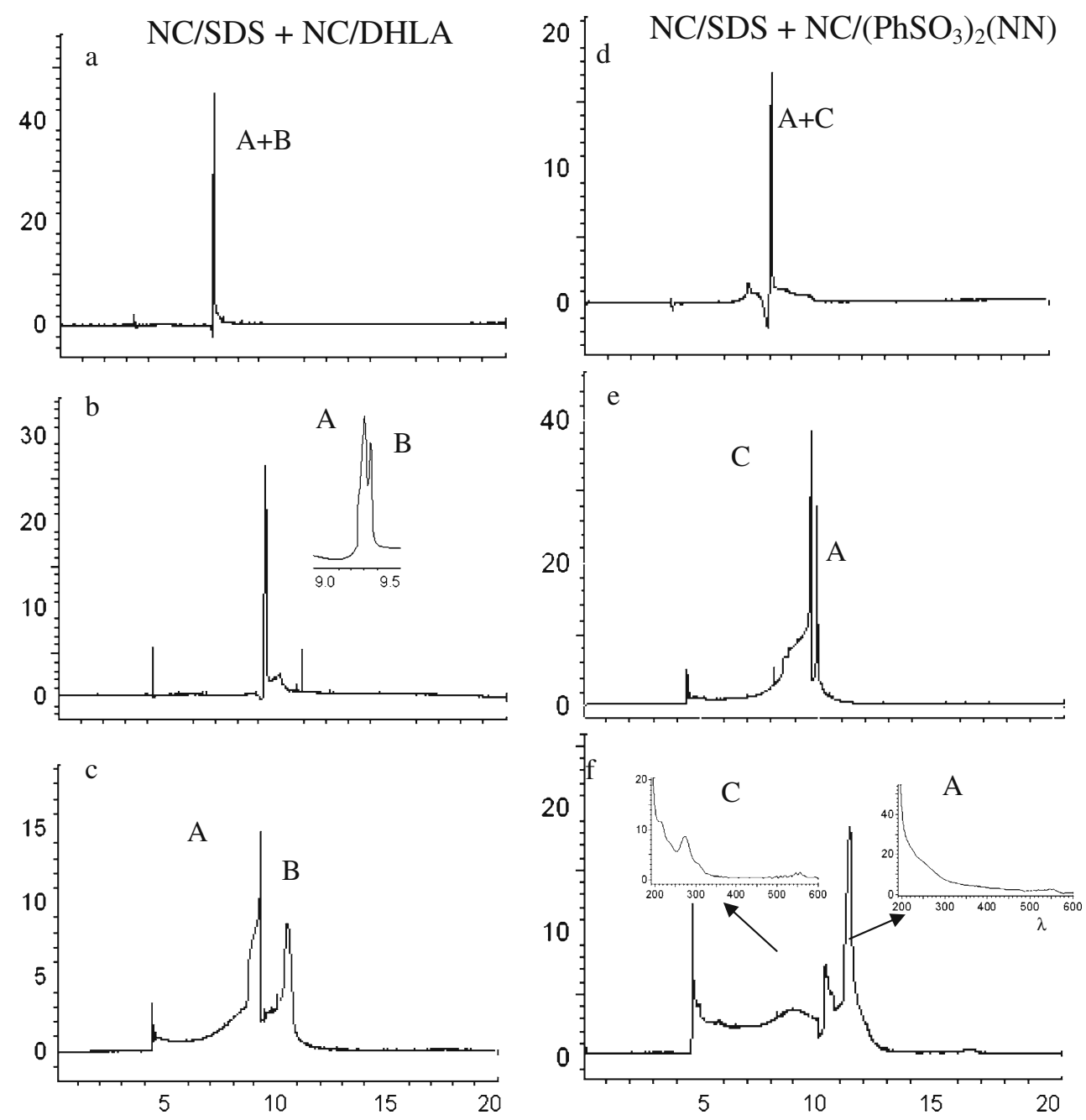

Time/ min.

(electropherogram b), the focusing effect was still observed and partial resolution of the nanocrystals was observed as shown in the inset. Increasing $\mathrm{ACN}$ to $15 \%$ caused both nanocrystal structures to migrate outside the micellar plug (electropherogram c). As can be seen, although the focusing effect was essentially gone, the two nanostructures could easily be separated.

An additional example was demonstrated for separating $\mathrm{CdSe} / \mathrm{ZnS} / \mathrm{TOPO}$ and its ligand-exchange product, $\mathrm{CdSe} /$ $\mathrm{ZnS} /\left(\mathrm{PhSO}_{3}\right)_{2}(\mathrm{NN})$, as shown in electropherograms d-f of Fig. 5. Like before, a bilayer was formed with an SDS surfactant on the $\mathrm{CdSe} / \mathrm{ZnS} / \mathrm{TOPO}$ surface to provide solubility for aqueous $\mathrm{CE}$ conditions. The $\mathrm{CdSe} / \mathrm{ZnS} /\left(\mathrm{PhSO}_{3}\right)_{2}(\mathrm{NN})$ $\mathrm{NCs}$ can be easily detected due to characteristic spectra being a superposition of both spectra (nanocrystal and ligand (NN); in agreement with ref. [45]). Similar to the results above, for DHLA-modified NCs, $0 \%$ ACN modifier in the $10 \mathrm{mM}$ sodium tetraborate separation buffer resulted in co-migration of both NCs within micellar plug. However, it was found that at $15 \% \mathrm{ACN}$, the distribution of nanocrystals between micellar/micelle-free zone, thus a focusing peak, as well as a peak for NCs being in the electrolyte buffer, were obtained (electropherogram e). At 20\% ACN modifier, loss of the NC focusing was observed as the NCs migrated outside the micellar zone into the micelle-free buffer zone.

The experiment above shows that a release of functionalized nanocrystals from a micellar plug can be controlled and one of factor for this is $\zeta$-potential of an apparent nanocrystal. Zeta potential measurements showed values of $-116 \mathrm{mV}$ for CdSe/ZnS/DHLA and $-7 \mathrm{mV}$ for CdSe/ $\mathrm{ZnS} /\left(\mathrm{PhSO}_{3}\right)_{2}(\mathrm{NN}) \mathrm{NCs}$, and reason for this can be a much extensive coverage of CdSe crystal surface with DHLA than with bulkier $\left(\mathrm{PhSO}_{3}\right)_{2}(\mathrm{NN})$ ligand. For $\mathrm{CdSe} / \mathrm{TOP} / /$ SDS NCs the $\zeta$-potential $(<-25 \mathrm{mV})$ was estimated [30]. To conclude both experiments above (Fig. 5), it can be stated that an electrolyte buffer modification does change affinity of NCs to a micellar plug, which regulates the NCs release from micellar to micelle-free zone. Moreover, the 
effect can be controlled and relies on the $\zeta$-potential of the charged particles.

An extension of the present section is the Electronic supplementary material, where CE separation of test molecules and test NCs was presented. In this section, it was indicated that a micellar plug implemented into separation of NCs, may play a double role: as a tool for regulating migration selectivity for NCs (Fig. S5 in the Electronic supplementary material) and as tool for 1-fold preconcentration of NCs in terms of $\mathrm{SEF}_{\text {height }}$ (Fig. S6 in the Electronic supplementary material). As test nanocrystals, amine-EviTag NCs were applied, that were used in the section below.

\section{Capillary electrophoresis of NCs modified with DNA}

Several current bioassays involve the use of biomolecules such as proteins and DNA attached to particles surfaces [2, 3]. However, only limited methods are available for measuring the degree of particle functionalization with biological ligands and the solution behavior of the biomolecule-nanoparticle conjugate. Thus far, most studies have utilized agarose gel electrophoresis to indicate binding of DNA to the particles surfaces, where the negative charge of DNA provides electrophoretic mobility proportional to the surface coverage [9-11]. A few CE separations of NCs functionalized with biological ligands have been reported $[17,18,46-48]$. In those examples, separations were mainly demonstrated for NCs before or after conjugation to large proteins using the CZE mode of separation.

Here we demonstrate the applicability of focusing mode for separating commercial amine-EviTags NCs functionalized with a DNA oligomer, thiol-terminated 20-mer (Fig. S7 in the Electronic supplementary material). Using procedures described in the Experimental section, the oligomer was attached to the amine-EviTag NCs and purified. The first example for this NCs was already shown in Figs. S5 and S6 in the Electronic supplementary material, where the migration of test molecule, free DNA oligomer, or amine-EviTags NCs, was shown to be manipulated by the introduced micellar zone.

Firstly, in order to compare, the sample containing amino-NCs starting material and oligomer-NC product was first separated by agarose gel electrophoresis using a previously published method [9-11]. The results (see Fig. S7(frame a) in the Electronic supplementary material) showed that the amino-NCs had a slight migration towards the cathode while the oligomer-NCs possessed substantial mobility towards the anode due to the oligomer polyanion. The lower electrophoretic mobility of amine-EviTags NCs was likely due to relatively low coverage with the primary amine ligands (ca. 30\% according to manufacturer).

The mixture of amine-EviTag NCs starting material and oligomer-NC product was next separated by CE. Although several conditions were tested, including one reported recently [18], traditional zone (CZE) and micellar (MEKC) proved to be unsuccessful in separating both nanocrystals and only one cumulative peak for both NCs was obtained. However, as shown in electropherogram of Fig. S7b (Electronic supplementary material), using CE separation mode with the micellar plug, it was possible to resolve the mixture of both NCs. The separation buffer contained $10 \mathrm{mM}$ sodium tetraborate and $15 \% \mathrm{ACN}$ as a modifier. The separation was conducted at $+20 \mathrm{kV}$. By tuning the separation conditions, a mixture of the amine-EviTags NCs starting material and the oligomer-modified NCs product were resolved. By observing the online UV-vis spectra (inset), the oligomer-NCs conjugate migrated within the micellar plug, whereas the amine-EviTags NCs migrated outside the micellar plug. This is an interesting result, which shows that despite the negative charge for oligomerNCs conjugate, as proven by agarose gel electrophoresis (Fig. S7a in the Electronic supplementary material), these NCs were migrated here as focused peak (Fig. S7b in the Electronic supplementary material). On the other hand, electrically neutral NCs were forced to exit a micellar zone (Fig. S7b in the Electronic supplementary material), which is a contradiction to the previous statement, that $\zeta$-potential controls release of the surface modified NCs from a micellar plug to a micelle-free zone.

It can be stated, that volume of a surface ligand attached to $\mathrm{NC}$ can be a decisive factor for this and two options should be considered. Firstly, for nanoparticles with less volumetric surface ligands, the distribution of NCs between micellar and micelle-free zone relies on $\mathrm{NC} \zeta$-potential, as seen in the section above. Secondly, this could change, considering NCs coated with bulkier ligands, based in the example reported in literature (the nonspecific wrapping of ligands around the particle (e.g., DNA [49]). In this case, the nanoparticles coated with bulker ligands were, structurally and behaviorally, similar to regular micelles. Note that for nanocrystals coated with ligands of different volumes, nanocrystals hydrodynamic radii do not follow the volume of a surface ligand, which means that conformation of a ligand around a particle (coiled or stretched) regulates the overall volume of a particle [50]. Thus, the migration of oligomer-NCs within a micellar zone, which led to NCs focusing, should be considered in terms of formation of the mixed pseudomicellar system with pseudomicelles (NCs wrapped by bulky ligands) and regular micelles. This allows these NCs to form more stable phase (mixed pseudomicellar system) compared with NCs coated with less volumetric ligands. To summarize, for this system, the NCs release from the phase (or generally choice between preconcentration/separation mechanisms) is controlled by a volume of a surface ligand attached to a nanostructure and the separation conditions applied. 
Capillary electrophoresis of silica-encapsulated NCs modified with DNA

Electrophoretic separations were also conducted on CdSe/ $\mathrm{ZnS}$ NCs encapsulated in a thin shell of functionalized silica. The surface of the nanostructures was functionalized by incorporating aminopropyl- and methylphosphonatesilane monomer at the end of the polymerization (Fig. S8 in the Electronic supplementary material). Amino functionality provides reactivity for binding to the thiol-terminated oligomer used here, while methylphosphonate enhanced water solubility. Dynamic light scattering and TEM results indicated the diameter of the NC/silica shell NPs to be $14 \pm$ $2 \mathrm{~nm}$ (Fig. 6). Zeta potential was measured to be $-80 \mathrm{mV}$, which is in agreement with the study of Bulavchenko and Popovetsky [51], where for $\mathrm{SiO}_{2}$ particles, using the electrophoretic measurement, value of $-73 \mathrm{mV}$ was obtained. Initially, plain gel electrophoresis was used to monitor the binding of DNA oligomer to the surface of $\mathrm{NC} /$ silica shell NPs as described above. However, unlike the results above, plain gel electrophoresis was unable to distinguish between modified and non-modified $\mathrm{NC} /$ silica shell NPs (see Fig. S8 in the Electronic supplementary material). Ran separately, both NPs migrated toward the anode. Thus, unlike previous case, with commercial aminoEviTags NCs, plain gel electrophoresis was unable to separate NC/silica shell NPs from NC/silica shell NPs modified with oligomer. Similar results were obtained when using recently published capillary electrophoresis methods for separating bioconjugated nanostructures $[18,46]$, and despite the peak visualization for individual NPs, no peaks resolution between these two nanoparticles were seen (one cumulative peak was seen, see example Fig. S9 in the Electronic supplementary material). This was likely due to the substantial negative surface charge from methylphosphonate functionality and silanol ionization on the NPs surface and further co-migration of both NPs.

However, as shown in the electropherogram of Fig. 6. the elaborated here conditions were able to successfully separate NC/silica shell NPs from NC/silica shell NPs modified with DNA oligomer. To the sample of NPs the micellar solution (TX-100/DOSS) was added, according to Procedures Section, in order to develop a micellar plug during a CE run. In this case, two peaks for NPs were obtained in the separation and identified by the online UVvis data as oligo-modified NC/silica shell NPs and unmodified NC/silica shell NPs. Under these conditions, the oligomer-NPs migrated and focused at the trailing edge of the micellar sample zone, while unmodified NPs migrated into the buffer zone. This is similar separation situation as for amino-NCs starting material and oligomer$\mathrm{NC}$ product, discussed in Section above. Both experiments confirm that the NPs distribution is directly related to
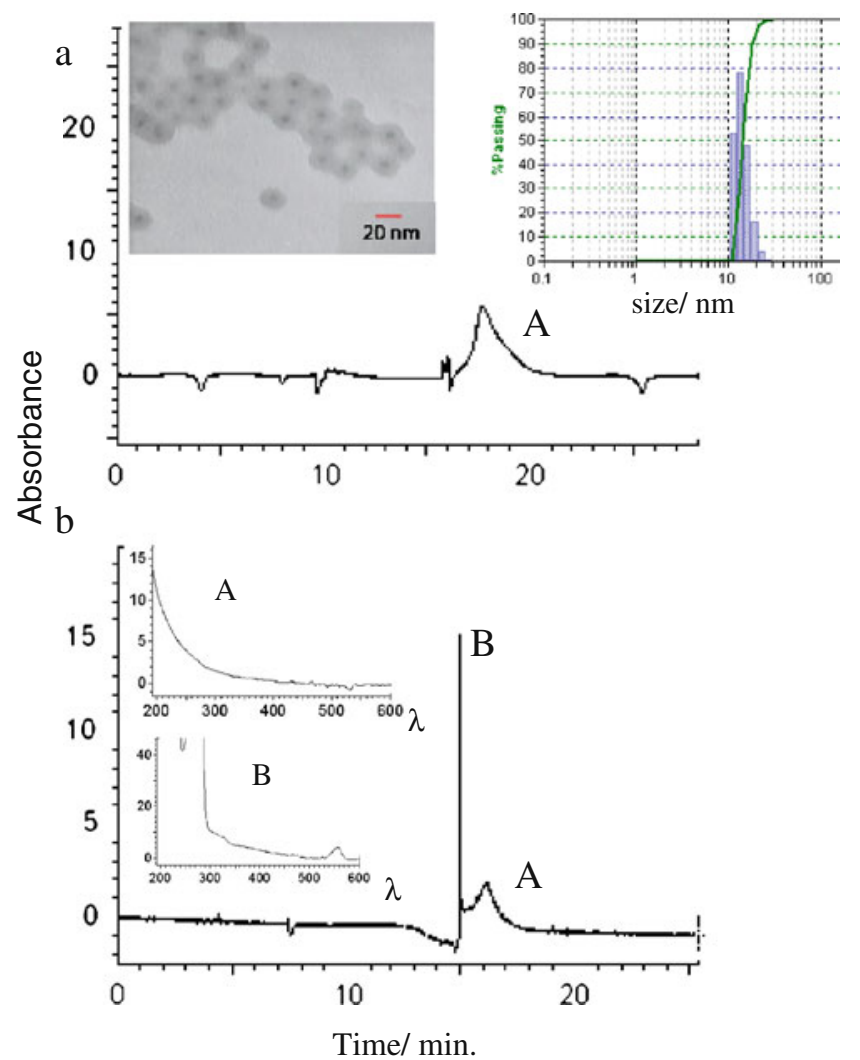

Fig. 6 Electrophoretic separation of silica-encapsulated $\mathrm{CdSe} / \mathrm{ZnS}$ NPs and its bioconjugated with aptamer form: a CdSe/ZnS silicaencapsulated NPs; b sample obtained after bioconjugation with aptamer and SEC purification. Electropherograms: a- $A, \mathrm{NC} /$ silica NPs, $\mathbf{b}-A, \mathrm{NC} /$ silica NPs; $B, \mathrm{NC} /$ silica/aptamer NPs. Samples: $\mathbf{a}$ the concentration NC/silica NPs in sample, based on UV-vis spectra is $2.5 \mu \mathrm{M}$; solution injected into capillary was a mixture (sample/ surfactant $=1: 3)$; $\mathbf{b}$ total $\mathrm{NCs}$ concentration $(\mathrm{NC} /$ silica $+\mathrm{NC} /$ silica/ aptamer) in sample based on UV-vis spectra is $\sim 0.83 \mu \mathrm{M}$; solution taken to $\mathrm{CE}$ analysis (sample/surfactant $=1: 1$ ). Composition of surfactant solution, $0.5 \mathrm{~g}$ TX-100 $(10 \% \mathrm{w} / \mathrm{w}$, water solution) was mixed with $0.07 \mathrm{~g}$ DOSS. Conditions: electrolyte buffer, $10 \mathrm{mM}$ sodium tetraborate; voltage: $10 \mathrm{kV}$, detection wavelength $293 \mathrm{~nm}$. a (insets) Examples of determination of the size of the synthesized $\mathrm{CdSe} / \mathrm{ZnS} /$ silica shell NPs by TEM image (left) and particle size analysis (right). Both methods point at average size of silicaencapsulated NCs equal to $14( \pm 2) \mathrm{nm}$

binding phenomena for nanoparticles/micellar zone system, due to the formation of mixed pseudomicellar system, where NPs coated with bulky ligands can serve as pseudomicelles. For the experiment with $\mathrm{NC} /$ silica shell NPs (Fig. 6), the ratio of both NPs is equal to the ratio of peak areas A to B, as only core of NPs $(\mathrm{CdSe} / \mathrm{ZnS})$ determines the particles optical properties. This allows us to estimate the bioconjugation efficiency of $23 \%$. Note that two forms of nanoparticles were detected simultaneously indicating good data reproducibility. However, the precision, in terms of, e.g., measurement of peak area, follows the order: focused NCs within a plug $>$ NCs being outside the plug, as the former is one-step process (preconcentra- 
tion), whereas the later is two-step process (preconcentration/release).

\section{Conclusions}

Results obtained in the present work show that an implementation of a micellar plug, is well-suited as an analytical tool for separating and characterizing NCs in solution. It was proved that, using NCs modified with a range of surface ligands, effects: shifting in migration of $\mathrm{NCs}$, preconcentration/separation mechanism for $\mathrm{NCs}$, are universal features in this method, irrespective of nanostructures examined. Based on several examples, it was pointed out that using CE mode with a micellar plug, the distribution of nanostructures between a micellar and a micelle-free zone, regulated by NCs affinity to micellar phase, is the controlling and decisive factor. Thus, the $\mathrm{CE}$ run of NCs according to a selected mechanism or due to double mechanism is available, and this was proved based on examples: test molecule/test NCs, as well as two test NCs. Due to developed here method, commercial amine-EviTags, with and without DNA oligomer attached to the nanocrystal surface, were clearly separated. Agarose gel electrophoresis confirmed this result. However, agarose gel and capillary zone electrophoresis could not resolve NC/silica shell NPs from NC/silica/ aptamer NPs, due to the relatively large charge inherent to the silica shell. The developed in this work method was able to separate both those NPs to confirm binding of DNA to the NPs surface and due to this the yield of the bioconjugation could be estimated. The separation procedures developed here allow for improving bioconjugation, as well as the product purification procedures, where nanostructures are involved.

Acknowledgments This study was supported by grant nos. FA955006-1-0365 and N 204142837 from the US Air Force Research Laboratory and Air Force Office of Scientific Research and the Ministry of Science and Higher Education (Poland), respectively.

Open Access This article is distributed under the terms of the Creative Commons Attribution Noncommercial License which permits any noncommercial use, distribution, and reproduction in any medium, provided the original author(s) and source are credited.

\section{References}

1. Michalet X, Pinaud FF, Bentolila LA, Tsay JM, Doose S, Li JJ, Sundaresan G, Wu AM, Gambhir SS, Weiss S (2005) Quantum dots for live cells, in vivo imaging, and diagnostics. Science 307:538-544

2. De M, Ghosh PS, Rotello VM (2008) Applications of nanoparticles in biology. Adv Mater 20:4225-4241
3. Medintz IL, Uyeda HT, Goldman ER, Mattoussi H (2005) Quantum dot bioconjugates for imaging, labelling and sensing. Nat Mater 4:435-446

4. Somers RC, Bawendi MG, Nocera DG (2007) CdSe nanocrystal based chem-/bio-sensors. Chem Soc Rev 36:579-591

5. Frasco MF, Chaniotakis N (2010) Bioconjugated quantum dots as fluorescent probes for bioanalytical applications. Anal Bioanal Chem 396:229-240

6. Murray CB, Kagan CR, Bawendi MG (2000) Synthesis and characterization of monodisperse nanocrystals and close-packed nanocrystal assemblies. Annu Rev Mater Sci 30:545-610

7. Klimov VI (2007) Spectral and dynamical properties of multiexcitons in semiconductor nanocrystals. Annu Rev Phys Chem 58:635-673

8. Seker F, Meeker K, Kuech TF, Ellis AB (2000) Surface chemistry of prototypical bulk II-VI and III-V semiconductors and implications for chemical sensing. Chem Rev 100:2505-2536

9. Parak WJ, Gerion D, Zanchet D, Woerz AS, Pellegrino T, Micheel Ch, Williams SC, Seitz M, Bruehl RE, Bryant Z, Bustamante C, Bertozzi CR, Alivisatos AP (2002) Conjugation of DNA to silanized colloidal semiconductor nanocrystalline quantum dots. Chem Mater 14:2113-2119

10. Gerion D, Pinaud F, Williams SC, Parak WJ, Zanchet D, Weiss S, Alivisatos AP (2001) Synthesis and properties of biocompatible water-soluble silica-coated $\mathrm{CdSe} / \mathrm{ZnS}$ semiconductor quantum dots. J Phys Chem B 105:8861-8871

11. Zanchet D, Micheel ChM, Parak WJ, Gerion D, Alivisatos AP (2001) Electrophoretic isolation of discrete Au nanocrystal/DNA conjugates. Nano Lett 1:32-35

12. Radko SP, Chrambach A (2002) Separation and characterization of sub- $\mu \mathrm{m}$ - and $\mu \mathrm{m}$-sized particles by capillary zone electrophoresis. Electrophoresis 23:1957-1972

13. Rodriguez MA, Armstrong DW (2004) Separation and analysis of colloidal/nano-particles including microorganisms by capillary electrophoresis: a fundamental review. J Chromatogr B $800: 7-25$

14. Liu F-K (2009) Analysis and applications of nanoparticles in the separation sciences A case of gold nanoparticles. J Chromatogr A 1216:9034-9047

15. Surugau N, Urban PL (2009) Electrophoretic methods for separation of nanoparticles. J Sep Sci 32:1889-1906

16. Pyell U (2008) CE characterization of semiconductor nanocrystals encapsulated with amorphous silicium dioxide. Electrophoresis 29:576-589

17. Pereira M, Lai EPC, Hollebone B (2007) Characterization of quantum dots using capillary zone electrophoresis. Electrophoresis 28:2874-2881

18. Vicente G, Coloń LA (2008) Separation of bioconjugated quantum dots using capillary electrophoresis. Anal Chem 80:1988-1994

19. Pyell U, Bücking W, Huhn C, Herrmann B, Merkoulov A, Mannhardt J, Jungclas H, Nann T (2009) Calibration-free concentration determination of charged colloidal nanoparticles and determination of effective charges by capillary isotachophoresis. Anal Bioanal Chem 395:1681-1691

20. Lin K-H, Chu T-C, Liu F-K (2007) On-line enhancement and separation of nanoparticles using capillary electrophoresis. J Chromatogr A 1161:314-321

21. Liu F-K (2007) A high-efficiency capillary electrophoresis-based method for characterizing the sizes of $\mathrm{Au}$ nanoparticles. J Chromatogr A 1167:231-235

22. Liu F-K (2009) Using micellar electrokinetic chromatography for the highly efficient preconcentration and separation of gold nanoparticles. J Chromatogr A 1216:2554-2559

23. Delgado AV, González-Caballero F, Hunter RJ, Koopal LK, Lyklema J (2007) Measurement and interpretation of electrokinetic phenomena. J Colloid Interface Sci 309:194-224 
24. Dubertret B, Skourides P, Norris D, Noireaux V, Brivanlou AH, Libchaber A (2002) In vivo imaging of quantum dots encapsulated in phospholipid micelles. Science 298:1759-1762

25. Fan H, Leve EW, Scullin Ch, Gabaldon J, Tallant D, Bunge S, Boyle T, Wilson MC, Brinker CJ (2005) Surfactant-assisted synthesis of water-soluble and biocompatible semiconductor quantum dot micelles. Nano Lett 5:645-648

26. Zhelev Z, Ohba H, Bakalova R (2006) Single quantum dotmicelles coated with silica shell as potentially non-cytotoxic fluorescent cell tracers. J Am Chem Soc 128:6324-6325

27. Bakalova R, Zhelev Z, Aoki I, Ohba H, Imai Y, Kanno I (2006) Silica-shelled single quantum dot micelles as imaging probes with dual or multimodality. Anal Chem 78:5925-5932

28. Jin T, Fujii F, Yamada E, Nodasaka Y, Kinjo M (2006) Control of the optical properties of quantum dots by surface coating with calix[n]arene carboxylic acids. J Am Chem Soc 128:9288-9289

29. Oszwałdowski S, Vasicek L, Tonev A, Roberts KP (2007) Characterization of CdSe nanocrystals and their interaction with micellar aggregates by means of capillary electrophoretic techniques. Nanotech Conf Tech Proc 4:332-335

30. Oszwałdowski S, Zawistowska K, Grisby L, Roberts KP (2010) Capillary electrophoretic separation and characterizations of CdSe quantum dots. Cent Eur J Chem 8:806-819

31. Islam MF, Rojas E, Bergey DM, Johnson AT, Yodh AG (2003) High weight fraction surfactant solubilization of single-wall carbon nanotubes in water. Nano Lett 3:269-273, nano

32. Moore VC, Strano MS, Haroz EH, Hauge RH, Smalley RE, Schmidt J, Talmon Y (2003) Individually suspended singlewalled carbon nanotubes in various surfactants. Nano Lett 3: 1379-1382

33. Wenseleers W, Vlasov II, Goovaerts E, Obraztsova ED, Lobach AS, Bouwen A (2004) Efficient isolation and solubilization of pristine single-walled nanotubes in bile salt micelles. Adv Funct Mater 14:1105-1112

34. Doorn SK, Fields RE, Hu H, Hamon MA, Haddon RC, Selegue JP, Majidi V (2002) High resolution capillary electrophoresis of carbon nanotubes. J Am Chem Soc 124:3169-3174

35. Treubig JM, Brown PR (2000) Novel approach to the analysis and use of fullerenes in capillary electrophoresis. J Chromatogr A 873:257-267

36. Sueyoshi K, Kitagawa F, Otsuka K (2008) On-line sample preconcentration and separation technique based on transient trapping in microchip micellar electrokinetic chromatography. Anal Chem 80:1255-1262
37. Quirino JP, Haddad PR (2008) Online sample preconcentration in capillary electrophoresis using analyte focusing by micelle collapse. Anal Chem 80:6824-6829

38. Quirino JP (2008) Neutral analyte focusing by micelle collapse in micellar electrokinetic chromatography. J Chromatogr A 1214:171-177

39. Quirino JP, Haddad PR (2009) Neutral analyte focusing by micelle collapse in partial-filling MEKC with UV and ESI-MS detection. Electrophoresis 30:1670-1674

40. Aranas AT, Guidote AM, Quirino JP (2009) Sweeping and new on-line sample preconcentration techniques in capillary electrophoresis. Anal Bioanal Chem 394:175-185

41. Quirino JP (2009) Analyte focusing by micelle collapse in CZE: Nanopreparation of neutrals. Electrophoresis 30:875-882

42. Armstrong DW, Girod M, He L, Rodriguez MA, Wei W, Zheng J, Yeung ES (2002) Mechanistic aspects in the generation of apparent ultrahigh efficiencies for colloidal (microbial) electrokinetic separations. Anal Chem 74:5523-5530

43. Evident Technologies. Available at: http://www.evidenttech.com

44. Landers JP (1997) Handbook of Capillary Electrophoresis. CRC Press, Danvers

45. Schmelz O, Mews A, Basche T, Herrmann A, Müllen K (2001) Supramolecular complexes from CdSe nanocrystals and organic fluorophors. Langmuir 17:2861-2865

46. Huang X, Weng J, Sang F, Song X, Cao Ch, Ren J (2006) Characterization of quantum dot bioconjugates by capillary electrophoresis with laser-induced fluorescent detection. J Chromatogr A 1113:251-254

47. Weng J, Song X, Li L, Qian H, Chen K, Xu X, Cao Ch, Ren J (2006) Highly luminescent CdTe quantum dots prepared in aqueous phase as an alternative fluorescent probe for cell imaging. Talanta 70:397-402

48. Feng H-T, Law W-S, Yu L-J, F-Y Li S (2007) Immunoassay by capillary electrophoresis with quantum dots. J Chromatogr A 1156:75-79

49. Parak WJ, Pellegrino T, Micheel ChM, Gerion D, Williams SC, Alivisatos AP (2003) Conformation of oligonucleotides attached to gold nanocrystals probed by gel electrophoresis. Nano Lett 3:33-36

50. Pons T, Uyeda HT, Medintz IL, Mattoussi H (2006) Hydrodynamic dimensions, electrophoretic mobility, and stability of hydrophilic quantum dots. J Phys Chem B 110:20308-20316

51. Bulavchenko AI, Popovetsky PS (2010) Electrokinetic potential of nanoparticles in reverse AOT micelles: photometric determination and role in the processes of heterocoagulation, separation, and concentration. Langmuir 26:736-742 